\title{
Impact of brief exposure to antifungal agents on the post-antifungal effect and hemolysin activity of oral Candida albicans
}

Arjuna Nishantha ELLEPOLA ${ }^{1}$, Rana KHAJAH ${ }^{1}$, Sumedha JAYATILAKE ${ }^{2}$, Lakshman SAMARANAYAKE ${ }^{3}$, Prem SHARMA $^{4}$, Zia KHAN ${ }^{5}$

\author{
1- Faculty of Dentistry, Health Sciences Center, Kuwait University, Kuwait. \\ 2- Faculty of Dental Sciences, University of Peradeniya, Sri Lanka. \\ 3- School of Dentistry, University of Queensland, Brisbane, Australia \\ 4- Faculty of Medicine, Health Sciences Center, Kuwait University, Kuwait. \\ 5- Department of Microbiology, Faculty of Medicine, Health Sciences Center, Kuwait University, Kuwait.
}

Corresponding address: Arjuna Ellepola - Department of Bioclinical Sciences - Faculty of Dentistry - Kuwait University - P.O. Box 24923 - Safat 13110 Kuwait - Phone: 0096524636714 - Fax: 0096525326049 - e-mail: arjuna@hsc.edu.kw

Submitted: March 11, 2015 - Modification: May 23, 2015 - Accepted: June 25, 2015

\section{ABSTRACT}

\begin{abstract}
Dost-antifungal effect (PAFE) of Candida and its production of hemolysin are determinants of candidal pathogenicity. Candida albicans is the foremost aetiological agent of oral candidosis, which can be treated with polyene, azole, and echinocandin antifungals. However, once administered, the intraoral concentrations of these drugs tend to be subtherapeutic and transient due to the diluent effect of saliva and cleansing effect of the oral musculature. Hence, intra-orally, Candida may undergo a brief exposure to antifungal drugs. Objective: Therefore, the PAFE and hemolysin production of oral C. albicans isolates following brief exposure to sublethal concentrations of the foregoing antifungals were evaluated. Material and Methods: A total of $50 \mathrm{C}$. albicans oral isolates obtained from smokers, diabetics, asthmatics using steroid inhalers, partial denture wearers and healthy individuals were exposed to sublethal concentrations of nystatin, amphotericin B, caspofungin, ketoconazole and fluconazole for $60 \mathrm{~min}$. Thereafter, the drugs were removed and the PAFE and hemolysin production were determined by previously described turbidometric and plate assays, respectively. Results: Nystatin, amphotericin $B$, caspofungin and ketoconazole induced mean PAFE (hours) of 2.2, 2.18, 2.2 and 0.62 , respectively. Fluconazole failed to produce a PAFE. Hemolysin production of these isolates was suppressed with a percentage reduction of $12.27,13.47,13.33,8.53$ and 4.93 following exposure to nystatin, amphotericin $B$, caspofungin, ketoconazole and fluconazole, respectively. Conclusions: Brief exposure to sublethal concentrations of antifungal drugs appears to exert an antifungal effect by interfering with the growth as well as hemolysin production of $C$. albicans.
\end{abstract}

Keywords: Candida albicans. Post-antifungal effect. Hemolysins. Antimycotics.

\section{INTRODUCTION}

Multiple physiognomies of Candida species have been suggested as virulence factors that enable the organism to cause superficial as well as disseminated infections in susceptible hosts ${ }^{11}$. Hemolysins are known to be critical virulent determinants contributing to candidal pathogenesis ${ }^{12}$. In particular, the secretion of hemolysin, followed by lysis of erythrocytes and subsequent acquisition of iron by Candida, facilitates hyphal invasion that contributes to the recalcitrance of the disease $\mathrm{e}^{12,13}$. In Candida albicans, it has been shown that secretion of hemolytic factors causes the release of hemoglobin, which is then used as an iron source by the organism ${ }^{13}$. Others have proposed that hemoglobin is an important host factor that triggers a different metabolic pathway necessary for establishing disseminated infections by $C$. albicans ${ }^{14}$. For instance, adding hemoglobin to defined media facilitates binding and adhesion of the yeast to several host proteins. This activity 
of hemoglobin is independent of iron acquisition, and is facilitated by a cell surface hemoglobin receptor. Furthermore, hemoglobin rapidly induces the expression of several genes, one of which, a heme oxygenase, allows the pathogen to consume exogenous heme or hemoglobin to acquire iron and produce the cytoprotective molecules ${ }^{14}$. Such exquisite recognition and responses to hemoglobin appear to be a unique adaptation of $C$. albicans to be a versatile opportunistic pathogen in the human host $^{14}$.

C. albicans is by far the leading fungal pathogen of oral candidosis, the commonest human fungal infection with a variety of clinical manifestations $^{9}$. Interestingly, more than $90 \%$ of human immunodeficiency virus (HIV) infected individuals develop oral candidosis during some point of their disease which is arguably the commonest oral manifestation in such patients. In addition, C. albicans has also been implicated in oral candidosis in other patient groups such as diabetics, asthmatics using inhalation steroids, smokers and denture wearers ${ }^{10,16,17}$. Recent studies have also shown that $C$. albicans isolates from subgingival biofilms of immunocompromised patients also produce hemolysins which may also exacerbate periodontal disease in these patients by insidiously enhancing the inflammatory process and tissue destruction ${ }^{15}$.

Growth suppression of Candida that occurs subsequent to limited exposure to antifungal agents, as in the case of the oral environment, has been described as the post-antifungal effect (PAFE). It has been proposed that the knowledge of PAFE would be clinically useful in evaluating new dosage regimens of a drug, and such curtailment of growth following transient exposure to antifungal agents may be a determinant of candidal virulence itself, as this phenomenon may modulate the virulent attributes of the yeast Candida ${ }^{5}$.

A number of antifungal therapeutic agents are accessible for treatment of oral Candida infections. These include the two polyenes group agents, nystatin and amphotericin B, the echinocandin agent caspofungin and the azoles such as ketoconazole (an imidazole) and fluconazole (a triazole) ${ }^{9}$. Despite the availability of these pharmacological agents for the management of oral candidosis, failure of therapy and ensuing recalcitrant infection is not uncommon ${ }^{9}$. One reason for this may be the distinctive nature of the oral milieu where the diluent effect of saliva and the cleansing effect of the oral musculature tend to reduce the bioavailability of antifungal agents below that of the effective therapeutic concentrations. Hence, intra-orally, the pathogenic Candida may undergo a transient exposure to an antifungal agent on administration, and subsequently the drug concentration is likely to be subtherapeutic ${ }^{5,9}$. Yet, the impact of such therapeutic levels of antifungals leading to PAFE and hemolysin production of oral $C$. albicans isolates obtained from different susceptible patient groups to oral candidosis (i.e., diabetics, asthmatics using inhalation steroids, smokers and partial denture wearers) has not been studied hitherto.

Hence, the main aim of this study was to determine the PAFE induced by five different antifungal agents belonging to three distinct classes, polyenes (nystatin, amphotericin B), echinocandins (caspofungin), and azoles (ketoconazole and fluconazole) on 50 oral C. albicans isolates. The opportunity was also taken to evaluate candidal hemolysin production under identical conditions of drug exposure.

\section{MATERIAL AND METHODS}

\section{Organisms}

A total of 50 oral isolates of C. albicans obtained in a previous study from patients seeking treatment from the Kuwait University Dental Clinic were included in the current study (10 isolates each from smokers, diabetics, asthmatics using steroid inhalation, patients wearing partial acrylic dentures and healthy individuals) ${ }^{7}$. None of the patients from which the isolates were recovered had clinically visible oral candidosis. Initially, all Candida isolates were tested for germ tube formation. Thereafter the colony characteristics were observed using CHROMagar Candida medium (Becton Dickinson and Company, Sparks, USA) and identified by VITEK 2 yeast ID system (BioMérieux, France) as well as API 20C AUX yeast ID system (BioMérieux, Inc, Hazelwood, MO, USA).

\section{Antifungal agents and media}

Nystatin, amphotericin B, ketoconazole and fluconazole (Sigma, St. Louis, MO, USA) were dissolved in dimethylsulphoxide (DMSO). Caspofungin (Merck and Company Inc, Waterhouse Station, NJ, USA) was dissolved in sterile distilled water. These anti-Candida agents were prepared initially as $10,000 \mu \mathrm{g} / \mathrm{ml}$ and stored at $-20^{\circ} \mathrm{C}$ prior to each experiment as previously described ${ }^{4,5}$. It was thereafter suspended/diluted in the following medium during the exposure period of yeasts: RPMI (Roswell Park Memorial Institute) 1640 medium buffered with 0.165 M MOPS (3-( $N$-morpholino) propanesulfonic acid) containing L-glutamine and lacking sodium bicarbonate (Sigma, USA), dissolved in 1 liter of sterile distilled water and adjusted to a $\mathrm{pH}$ of 7.2 and sterilized filter.

\section{Determination of minimal inhibitory concentration (MIC)}

The susceptibility of the C. albicans isolates to 
antifungal agents was determined as described in an earlier study of ours ${ }^{7}$. The MIC values against amphotericin B, caspofungin, ketoconazole and fluconazole were determined by $E$ test, performed according to manufacturer's recommendations ( $A B$ BIODISK, Solna, Sweden). As depicted in previously freshly subcultured isolates (five colonies) were uniformly suspended in sterile saline, and turbidity was adjusted to 0.5 McFarland Standard. The McFarland Standard 0.5 is approximately equal to $1 \times 10^{6}$ to $5 \times 10^{6}$ cells $/ \mathrm{ml}^{3}$. This inoculum was swabbed onto the agar plates (150 $\mathrm{mm}$ diameter) and allowed to dry for 10-15 min before the $\mathrm{E}$ test strips were applied. RPMI 1640 agar supplemented with $2 \%$ glucose and buffered with MOPS $(0.165$ $\mathrm{M}$; $\mathrm{pH}$ 7.0) was used for susceptibility testing according to the method recommended by the CLSI (formerly National Committee for Clinical Laboratory Standards; M27-A2). The plates were incubated at $35^{\circ} \mathrm{C}$, and MIC was observed after 24-48 $\mathrm{h}$ of incubation. The point where inhibition ellipses intercepted the scale on the antifungal strip was taken as the MIC for each test isolate: complete inhibition (100\%) of growth for amphotericin B and caspofungin, and marked decrease in growth intensity $(80 \%)$ for fluconazole and ketoconazole. Reference strains of C. albicans, ATCC 90028, and C. parapsilosis, ATCC 22019, were used as reference strains. Interpretive susceptibility breakpoints for fluconazole were those recommended by CLSI document M27-A2. Due to the dearth of defined susceptibility breakpoints for amphotericin B and ketoconazole, an isolate was considered susceptible with an MIC breakpoint of $\leq 1.0 \mu \mathrm{g} /$ $\mathrm{ml}$ for amphotericin B and of $0.125 \mu \mathrm{g} / \mathrm{ml}$ for ketoconazole? ${ }^{7}$. For caspofungin, isolates with MIC of $\leq 2.0 \mu \mathrm{g} / \mathrm{ml}$ were considered as susceptible ${ }^{7}$.

As previously performed, the MIC values of nystatin were established by the broth dilution technique ${ }^{5}$ by performing two-fold serial dilutions of the drug in microtitre plates using an inoculum of $1-5 \times 10^{5}$ colony forming units (CFU) $/ \mathrm{ml}$. The MIC was determined visually following $24 \mathrm{~h}$ incubation at $37^{\circ} \mathrm{C}$. The MIC was defined as the lowermost concentration of the drug that impeded growth of Candida cells, as indicated by the absence of turbidity (optically clear). The MIC was read independently by a couple of laboratory personals. C. albicans ATCC 90028 was used as a reference strain.

\section{Preparation of cell suspension for the PAFE and hemolysin production assay}

A heretofore explained method was used for this purpose ${ }^{4-6}$. Briefly, Candida cells maintained on Sabouraud Dextrose Agar (SDA), were inoculated onto fresh plates and incubated overnight at $37^{\circ} \mathrm{C}$ for $24 \mathrm{~h}$ prior to use. The organisms were harvested and a cell suspension prepared in sterile phosphate buffered saline (PBS) at $520 \mathrm{~nm}$ to an optical density of 1.5 . From this cell suspension, $1 \mathrm{ml}$ was added to tubes containing $4 \mathrm{ml}$ of RPMI broth (control) and $4 \mathrm{ml}$ of RPMI/drug solution (test), in which the drug concentration was twice the MIC. This gave a cell suspension of approximately $10^{6}$ cells $\mathrm{ml}^{-1}$ in each assay tube. The tubes were then incubated at $37^{\circ} \mathrm{C}$ for a period of $60 \mathrm{~min}$. Following this limited exposure, the drugs were removed by two cycles of dilution with sterile PBS and centrifugation. Supernatant was thereafter completely decanted and the pellets were re-suspended in $2.5 \mathrm{ml}$ of sterile PBS. This technique has been used previously for drug removal, and has revealed to minimize the concentration of the drug as much as 10,000 fold, thus curtailing any carry-over effect of the drug following its removal ${ }^{3-5}$. Viable counts of the control and the test were performed after drug removal. As the procedure of drug removal effectively eliminated any carry-over effect, there was virtually no difference on the viable counts of the control and the tests following exposure to already diluted subtherapeutic concentrations of the drug, as observed in previous studies ${ }^{4-6}$.

\section{PAFE assay}

Following drug removal, in order to ascertain the growth suppression and ensue the recovery of fungal growth, namely the PAFE, the growth was determined by a previously described optical density method with minor amendments using the equation PAFE $=\mathrm{T}-\mathrm{C}^{4-6}$. $\mathrm{T}$ is the time required for optical density of the drug-exposed cell suspension to reach the selected relative optical density of 0.05 value at $520 \mathrm{~nm}$. C is the time required for optical density of the drug-free control cell suspension to reach the selected relative optical density value at $520 \mathrm{~nm}$. Thus, T-C expresses the time in which the antifungal agent was capable of causing growth suppression of the organism following limited exposure to the drug (i.e., PAFE). As in previous studies, $1600 \mu \mathrm{l}$ of cell suspension was incubated with $2.4 \mathrm{~mL}$ of RPMI 1640 at $37^{\circ} \mathrm{C}$ and the relative optical density of the suspension was measured at frequent intervals (i.e., $15 \mathrm{~min}$ ) for $8 \mathrm{~h}$ by which time, both the controls and tests reached the selected relative optical density value, enabling to calculate the PAFE ${ }^{4-6}$.

\section{Hemolysin assay}

Hemolysin production was determined with slight modifications using a plate assay previously described ${ }^{1,12,13}$. In short, $10 \mu$ l of the aforementioned treated antifungal and control suspensions were spot-inoculated on a blood agar supplemented with $3 \%$ glucose $(\mathrm{w} / \mathrm{v})$. The final $\mathrm{pH}$ of the medium was $5.6 \pm 0.2$. Spot-inoculation was performed to yield a 
circular inoculation site of about $10 \mathrm{~mm}$ in diameter. The plates were then incubated at $37^{\circ} \mathrm{C}$ in $5 \% \mathrm{CO}_{2}$ for $48 \mathrm{~h}$. After incubation during this period, the diameter of the colony (y) and the diameter of the colony plus the translucent zone of hemolysis $(x)$ were measured. The ratio of $x / y$, which represents the hemolytic activity (hemolytic index), was measured as done previously ${ }^{1,12,13}$.

All experiments were repeated on three separate occasions with duplicate determinations on each occasion.

\section{Statistical analysis}

For the suppression effect on hemolysin production, the raw data did not follow normal distribution. Therefore, in accordance to nonparametric statistician advices, Wilcoxon Signed Ranks test was used to compare the control group with each of the five groups exposed five different antifungal agents. The difference between the groups exposed to five different antifungal drugs were also compared using Wilcoxon Signed Ranks test in relation to drug-induced PAFE and suppression of hemolysin production. A $p$ value of less than 0.05 was considered statistically significant.

\section{RESULTS}

All C. albicans isolates were susceptible to the tested antifungal drugs. The MIC $(\mu \mathrm{g} / \mathrm{ml})$ values of all C. albicans isolates for nystatin obtained from the broth dilution technique ranged between 0.78-1.56. The MIC values obtained by E-tests for amphotericin $B$ were between 0.004-0.19, and for caspofungin it ranged between 0.004-0.125. Ketoconazole elicited a MIC of 0.004-0.032 while it ranged between 0.047-0.125 for fluconazole.
Based on the equation $\mathrm{PAFE}=\mathrm{T}-\mathrm{C}$, the mean in vitro PAFE (hours) on 50 oral isolates of $C$. albicans following $1 \mathrm{~h}$ exposure and subsequent removal of nystatin, amphotericin $B$, caspofungin and ketoconazole was 2.20, 2.18, 2.20 and 0.62 , respectively (Table 1 ). Hence, in summary, brief exposure to nystain, amphotericin B and caspofungin was able to suppress the growth of $C$. albicans isolates giving a PAFE of approximately $2 \mathrm{~h}$ whereas it was approximately $1 / 2 \mathrm{~h}$ for ketoconazole. Fluconazole failed to elicit a detectable PAFE.

Mean hemolysin production (hemolytic index) of the $50 \mathrm{C}$. albicans isolates, following limited exposure to five different sublethal concentrations of antifungal agents, drug removal and subsequent production of hemolysin production is shown in Table 2. Mean hemolysin production of the unexposed isolates was 1.50, whereas this figure following exposure to nystatin, amphotericin B, caspofungin, ketoconazole and fluconazole was 1.32, 1.30, 1.30, 1.37 and 1.42 , respectively. Hence, as shown in Table 2 and 3, a significant reduction in hemolysin activity was observed following the exposure to all of these drugs, with a mean percentage reduction of $12.27,13.47,13.33,8.53$ and 4.93 for nystatin, amphotericin B, caspofungin, ketoconazole and fluconazole, respectively $(p<0.001$ for all).

The analysis of the difference on PAFE and the suppressive effect on hemolysin production elicited by the five different antifungal agents revealed that the PAFE and the suppressive effect elicited by the polyene and echinocandin antifungal agents were significantly higher and statistically different from that of the two azole drugs $(p<0.001)$. In addition, the PAFE and the hemolysin suppressive effect elicited by the two azoles were also statistically different from each other $(p<0.001)$, with ketoconazole having a superior effect than

Table 1-The post-antifungal effect (PAFE) (in hours) of 50 oral C. albicans isolates following 1 hour exposure and subsequent removal of five different antifungal drugs. Each value indicates the mean (SD) of 10 oral $C$. albicans obtained either from healthy individuals, diabetic patients, asthmatic patients, smokers or denture wearers. Regarding PAFE, $0.25=15$ minutes; $0.50=30$ minutes; $0.75=45$ minutes; $1.0=60$ minutes. SD: Standard deviation. SEM: Standard error of mean

\begin{tabular}{cccccc}
\hline $\begin{array}{c}\text { Source of } \boldsymbol{C} \text {. albicans } \\
\text { isolates }(\mathrm{n}=50)\end{array}$ & Nystatin & Amphotericin B & Caspofungin & Ketoconazole & Fluconazole \\
\hline Healthy $(n=10)$ & $2.21(0.21)$ & $2.18(0.20)$ & $2.22(0.21)$ & $0.65(0.11)$ & $\begin{array}{c}\text { No detectable } \\
\text { change in growth } \\
\text { suppression }\end{array}$ \\
Diabetic $(n=10)$ & $2.17(0.21)$ & $2.10(0.19)$ & $2.20(0.20)$ & $0.60(0.09)$ \\
Asthmatic $(n=10)$ & $2.19(0.19)$ & $2.30(0.13)$ & $2.20(0.20)$ & $0.62(0.09)$ \\
Smokers $(n=10)$ & $2.20(0.20)$ & $2.22(0.21)$ & $2.21(0.19)$ & $0.63(0.10)$ \\
Denture Wearers $(n=10)$ & $2.22(0.22)$ & $2.12(0.21)$ & $2.16(0.18)$ & $0.60(0.09)$ \\
Mean $(n=50)$ & 2.2 & 2.18 & 2.2 & 0.62 \\
SEM & 0.02 & 0.02 & 0.02 & 0.002 \\
SD & 0.2 & 0.2 & 0.19 & 0.1 \\
\hline
\end{tabular}


Table 2- Mean (SD) hemolysin activity (hemolytic index) of 50 oral C. albicans isolates following 1 hour exposure and subsequent removal of five different antifungal drugs. Each value indicates the mean (SD) hemolysin activity of 10 oral $C$. albicans obtained either from healthy individuals, diabetic patients, asthmatic patients, smokers or denture wearers. SD: Standard deviation. SEM: Standard error of mean

\begin{tabular}{ccccccc}
\hline $\begin{array}{c}\text { Source of } \boldsymbol{C} \text {. } \\
\text { albicans isolates } \\
(\mathbf{n}=\mathbf{5 0})\end{array}$ & $\begin{array}{c}\text { Unexposed } \\
\text { controls }\end{array}$ & Nystatin & Amphotericin B & Caspofungin & Ketoconazole & Fluconazole \\
\hline Healthy & 1.49 & 1.31 & 1.29 & 1.29 & 1.37 & 1.42 \\
$(n=10)$ & -0.02 & -0.02 & -0.05 & -0.05 & -0.02 & -0.02 \\
Diabetic & 1.5 & 1.3 & 1.27 & 1.3 & 1.38 & 1.43 \\
$(n=10)$ & -0.03 & -0.03 & -0.06 & -0.05 & -0.03 & -0.02 \\
Asthmatic & 1.5 & 1.33 & 1.31 & 1.32 & 1.38 & 1.43 \\
$(n=10)$ & -0.02 & -0.05 & -0.07 & -0.06 & -0.03 & -0.03 \\
Smokers & 1.51 & 1.34 & 1.33 & 1.29 & 1.36 & 1.43 \\
$(n=10)$ & -0.02 & -0.06 & -0.07 & -0.07 & -0.02 & -0.04 \\
\hline Denture Wearers & 1.5 & 1.3 & 1.29 & 1.3 & 1.37 & 1.42 \\
$(n=10)$ & -0.03 & -0.07 & -0.08 & () $.05)$ & -0.03 & -0.02 \\
\hline Mean ( $=50)$ & 1.5 & 1.32 & 1.3 & 1.3 & 1.37 & 1.42 \\
SEM & 0.003 & 0.007 & 0.009 & 0.008 & 0.004 & 0.004 \\
SD & 0.02 & 0.05 & 0.06 & 0.06 & 0.03 & 0.03 \\
\hline$p$ value & & $p<0.001$ & $p<0.001$ & $p<0.001$ & $p<0.001$ & $p<0.001$ \\
\hline
\end{tabular}

Table 3- Summary of the mean percentage suppression of hemolysin activity in 50 oral C. albicans isolates following 1 hour exposure and subsequent removal of five different antifungal agents. Each value indicates the mean percentage reduction of hemolysin activity in 10 oral C. albicans obtained from either healthy individuals, diabetic patients, asthmatic patients, smokers or denture wearers. SD: Standard deviation. SEM: Standard error of mean

\begin{tabular}{cccccc}
\hline $\begin{array}{c}\text { Source of } \mathbf{C} \text {. albicans } \\
\text { Isolates }(\mathbf{n}=\mathbf{5 0})\end{array}$ & Nystatin & Amphotericin B & Caspofungin & Ketoconazole & Fluconazole \\
\hline Healthy & 12.08 & 13.42 & 13.42 & 8.05 & 4.7 \\
\hline Diabetic & 13.33 & 15.33 & 13.33 & 8 & 4.66 \\
\hline Asthmatic & 11.33 & 12.66 & 12 & 8 & 4.66 \\
\hline Smokers & 11.26 & 11.92 & 14.57 & 9.93 & 5.3 \\
\hline Denture Wearers & 13.33 & 14 & 13.33 & 8.66 & 5.33 \\
\hline Mean & 12.27 & 13.47 & 13.33 & 8.53 & 4.93 \\
\hline SEM & 0.46 & 0.58 & 0.41 & 0.37 & 0.16 \\
\hline SD & 1.02 & 1.3 & 0.91 & 0.83 & 0.35 \\
\hline
\end{tabular}

fluconazole. However, the PAFE and the suppressive effect on hemolysin production elicited by the two polyenes and the echinocandin agent were not significantly different from each other.

Finally, when comparing the clinical isolates from diabetics, asthmatics, smokers, denture wearers and healthy individuals, there were no difference in either PAFE or hemolysin production on exposure to sublethal concentrations of any of the antifungals.

\section{DISCUSSION}

C. albicans is by far the major aetiological agent of oral candidosis ${ }^{8}$. Hemolysin is one of C. albicans recently recognized, putative virulent factors. In particular, the secretion of hemolysin facilitates hyphal invasion, which is critical for disseminated candidal infection ${ }^{1,12-14}$. As described above, it is known that PAFE, the capacity of Candida to recover and grow after an antifungal-insult, is another important measure of the virulence of the yeast ${ }^{4-7}$. We, along with others, have shown that more 
virulent and resistant Candida will have a low PAFE, whereas susceptible and less virulent organisms will have higher PAFEs ${ }^{4-7}$.

The present results indicate that brief exposure to sublethal concentrations of nystatin, amphotericin $B$, caspofungin and ketoconazole elicited PAFEs ranging from approximately 30 to $120 \mathrm{~min}$ with the $C$. albicans isolates tested. A notable exception was fluconazole, which did not elicit a PAFE on the tested isolates. The observed PAFE may be related to the pharmacodynamics of these antimycotics on Candida. For instance, both polyenes (nystatin and amphotericin B) bind to the sterol components in the yeast cell wall and make it more penetrable ${ }^{5,9}$. Furthermore, investigations have shown internally collapsed cells with an intact cell wall leaving "ghosts-like" cells, and deflated Candida cells following exposure to subcidal concentrations of polyenes $^{8}$. Caspofungin, on the contrary, acts by inhibiting the enzyme responsible for biosynthesis of $\beta-1,3$ glucan resulting in disturbing the integrity of the fungal cell wall ${ }^{2}$. In addition, caspofungin has shown excellent growth-inhibitory activity against Candida at low concentrations ${ }^{2}$. As for the azoles (ketoconazole and fluconazole), they alter the fungal cell membranes by blocking the 14 a-demethylation step in the biosynthesis of ergosterol. The consequent depletion of ergosterol and accumulation of 14 a-methyl-sterols leads to alterations in a number of membrane-associated functions ${ }^{4,9}$. Considering the foregoing wide range pharmacodynamic properties of the agents we have studied, it is tempting to speculate that the structural changes of the cell wall, as well as the metabolic effects elicited by the drugs, would all contribute to the suppression of growth eliciting a PAFE. Surprisingly, fluconazole failed to elicit a detectable PAFE response in spite of this drug being remarkably more effective in vivo than ketoconazole in the management of candidal infection ${ }^{9}$. Interestingly, many workers have shown that fluconazole's growth inhibitory activity against C. albicans isolates in vitro is much less than that of ketoconazole $e^{4,9,18}$. Hence, the later could be one reason for the absence of detectable PAFE following exposure to fluconazole.

The exposure of $C$. albicans isolates to the foregoing drugs was also capable of suppressing hemolysin production of the yeast to significant varying levels ranging from $5 \%$ to $13.5 \%$. A previous single study has also shown a reduction of hemolysin activity of $C$. albicans, as well as Candida tropicalis isolates, following exposure to nystatin, amphotericin B and fluconazole ${ }^{1}$. It is rather difficult to fathom the exact mechanism by which these drugs modulate changes in hemolysin production in Candida species. However, taken collectively, the current findings contribute to the understanding of pharmacodynamics of these antifungal agents against two virulent determinants (i.e., PAFE and hemolysin production) incriminated in the pathogenesis of $C$. albicans infections. In addition, as hemolysins are known to mediate host invasion, particularly by facilitating hyphal penetration of the epithelium, it can be speculated that, by suppressing hemolysin production, these antifungals could also minimize yeast dissemination from and within the oral and periodontal tissues.

The significant difference in PAFE elicited by polyenes and echinocandins and azoles, as well as their suppressive effect on hemolysin production of Candida, as seen in this study, may well be due to the differences in the pharmacodynamics of the different antimycotic groups. Polyenes and echinocandins are fungicidal against $C$. albicans whereas azoles are fungistatic ${ }^{9}$. This could be the foremost explanation for the exquisite sensitivity of C. albicans to nystatin, amphotericin B and caspofungin compared to the azoles. In addition, we also noted a significant difference in the PAFE and hemolysin production when the yeasts were exposed to the two azoles. As previously mentioned, others have shown that the growth inhibitory activity of fluconazole against $C$. albicans in vitro is much less than that of ketoconazole $e^{4,9,18}$. These observations may be related to the fact that ketoconazole is an imidazole whereas fluconazole is a triazole. Arguably, therefore, the differences in the mode of activity between the two azoles may account for ketoconazole and fluconazole elicited variations in PAFE and hemolysin production.

To our knowledge, the current study is the first one to document the antifungal-induced PAFE using a wide array of antifungal agents and the suppression of hemolysin production during the PAFE period, covering the largest number $(n=50)$ of oral C. albicans isolates obtained from a single geographic locale. Testing with a larger number of sessile isolates encased in a Candida biofilm scenario instead of using only planktonic isolates as used in this study is warranted to further elucidate the current findings. The exact cellular mechanisms by which antifungals interfere with hemolysin activity in Candida also remain to be elucidated.

\section{CONCLUSIONS}

Brief and transient exposure to sublethal concentrations of antifungal drugs may induce a PAFE and modulate hemolysin production of oral C. albicans isolates, thereby adversely affecting their pathogenic potency. The data herein also provide an insight into the possible mechanisms of antifungal agents, at subtherapeutic concentrations, in biological milieus such as the oral cavity. 


\section{ACKNOWLEDGEMENTS}

The study was partially supported by Kuwait University Research Grant No. DB 01/14. The authors thankfully acknowledged the technical advice and support in laboratory procedures for carrying out this project given by Ms. Rachel Chandy and Ms. Preethi John of Health Sciences Center, Kuwait University, Kuwait.

\section{REFERENCES}

1- Anil S, Hashem M, Vellappally S, Patil S, Bandara HM, Samaranayake LP. Sub-inhibitory concentrations of antifungals suppress hemolysin activity of oral Candida albicans and Candida tropicalis isolates from HIV-infected individuals. Mycopathologia. 2014;178:207-15.

2- Bizerra FC, Melo AS, Katchburian E, Freymüller E, Straus $\mathrm{AH}$, Takahashi $\mathrm{HK}$, et al. Changes in cell wall synthesis and ultrastructure during paradoxical growth effect of caspofungin on four different Candida species. Antimicrob Agents Chemother. 2011;55:302-10.

3- Clinical and Laboratory Standards Institute. Method for antifungal disk diffusion susceptibility testing of yeasts: approved guideline M44-A. Wayne: NCCLS; 2004.

4- Ellepola AN, Chandy R, Khan ZU. Post-antifungal effect and adhesion to buccal epithelial cells of oral Candida dubliniensis isolates subsequent to limited exposure to amphotericin B, ketoconazole and fluconazole. J Investig Clin Dent. 2014;doi: 10.1111/jicd.12095. Epub ahead of print.

5- Ellepola AN, Joseph BK, Chandy R, Khan ZU. The postantifungal effect of nystatin and its impact on adhesion attributes of oral Candida dubliniensis isolates. Mycoses. 2014;57:56-63.

6- Ellepola AN, Joseph BK, Khan ZU. The postantifungal effect and phospholipase production of oral Candida albicans from smokers, diabetics, asthmatics, denture wearers and healthy individuals following brief exposure to subtherapeutic concentrations of chlorhexidine gluconate. Mycoses. 2014;57:553-9.
7- Ellepola AN, Khan ZU, Joseph BJ, Chandy R, Philip L. Prevalence of Candida dubliniensis among oral Candida isolates in patients attending the Kuwait University Dental Clinic. Med Princ Pract. 2011;20:271-6.

8- Ellepola AN, Samaranayake LP. The effect of limited exposure to antifungal agents on the germ tube formation of oral Candida albicans. J Oral Pathol Med. 1998;27:213-9.

9- Ellepola AN, Samaranayake LP. Oral candidal infections and antimycotics. Crit Rev Oral Biol Med. 2000;11:172-98.

10- Ellepola AN, Samaranayake LP. Inhalation and topical steroids, and oral candidosis: a mini review. Oral Dis. 2001;7:211-6.

11- Haynes K. Virulence in Candida species. Trends Microbiol. 2001;9:591-6.

12- Luo G, Samaranayake LP, Yau JY. Candida species exhibit differential in vitro hemolytic activities. J Clin Microbiol. 2001;39:2971-4.

13- Manns JM, Mosser DM, Buckley HR. Production of a hemolytic factor by Candida albicans. Infect Immun. 1994;62:5154-6.

14- Pendrak ML, Yan SS, Roberts DD. Sensing the host environment: recognition of hemoglobin by the pathogenic yeast Candida albicans. Arch Biochem Biophys. 2004;426:148-56.

15- Sardi JC, Duque C, Höfling JF, Gonçalves RB. Genetic and phenotypic evaluation of Candida albicans strains isolated from subgingival biofilm of diabetic patients with chronic periodontitis. Med Mycol. 2012;50:467-75.

16- Soysa NS, Ellepola AN. The impact of cigarette/tobacco smoking on oral candidosis: an overview. Oral Dis. 2005;11:26873.

17- Soysa NS, Samaranayake LP, Ellepola AN. Diabetes mellitus as a contributory factor in oral candidosis. Diabet Med. 2006;23:4559.

18- Troke PF, Marriott MS, Richardson K, Tarbit MH. In vitro potency and in vivo activity of azoles. Ann N Y Acad Sci. 1988;544:284-93. 